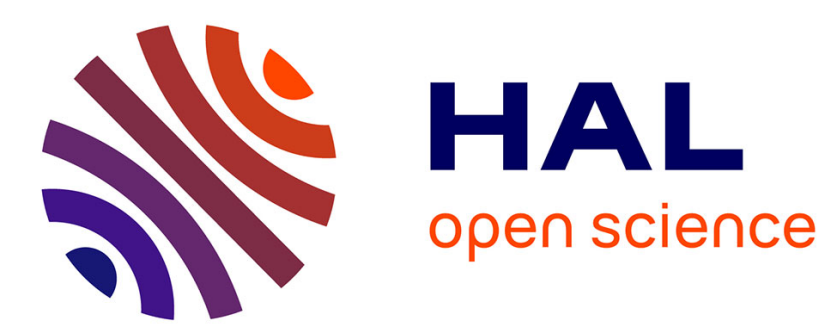

\title{
An enhanced 1-hop clustering algorithm for Publish / Subscribe systems in AANETS
}

Mickaël Royer, Fabien Garcia, Alain Pirovano

\section{To cite this version:}

Mickaël Royer, Fabien Garcia, Alain Pirovano. An enhanced 1-hop clustering algorithm for Publish / Subscribe systems in AANETS. DASC 2015 IEEE/AIAA 34th Digital Avionics Systems Conference, Sep 2015, Prague, Czech Republic. pp 2D2-1 - 2D2-6, ISBN: 978-1-4799-8939-3 10.1109/DASC.2015.7311373 . hal-01337738

\section{HAL Id: hal-01337738 \\ https://hal-enac.archives-ouvertes.fr/hal-01337738}

Submitted on 27 Jun 2016

HAL is a multi-disciplinary open access archive for the deposit and dissemination of scientific research documents, whether they are published or not. The documents may come from teaching and research institutions in France or abroad, or from public or private research centers.
L'archive ouverte pluridisciplinaire HAL, est destinée au dépôt et à la diffusion de documents scientifiques de niveau recherche, publiés ou non, émanant des établissements d'enseignement et de recherche français ou étrangers, des laboratoires publics ou privés. 


\title{
AN ENHANCED 1-HOP CLUSTERING ALGORITHM FOR PUBLISH / SUBSCRIBE SYSTEMS IN AANETS
}

\author{
Mickaël Royer, Fabien Garcia and Alain Pirovano \\ ENAC Toulouse, France
}

\begin{abstract}
Content-based Publish / Subscribe communication paradigm offers a new approach to disseminate messages in the network, where the message content determines the recipients. Many applications used on AANETs, which are a subclass of VANETs, could be more efficient using this paradigm. Many Publish / Subscribe systems suitable for VANETs have been developed, however they are not efficient for some AANET applications. A promising approach is to build a Publish / Subscribe system over a cluster structure to reduce the control overhead and to offer a good scalability. However, the efficiency of this approach strongly depends on the performance of the clustering algorithm. The aim of this article is to propose a new clustering method, named CAPS, which will be the basis for a future content-based Publish / Subscribe system for AANETs. To validate our approach, a simulation model has been developed. Our algorithm has been compared to some other solutions in a modeled AANET context based on real air traffic traces. We show that CAPS gives better results than other solutions in terms of stability while maintaining at a low level the number of cluster groups.

Index Terms-Vehicular Ad Hoc Network (VANET), Aeronautical Ad Hoc Network (AANET), Clustering Algorithm, Publish / Subscribe communication systems.
\end{abstract}

\section{Introduction}

The content-Based Publish / Subscribe paradigm offers many advantages over the classical communication paradigm. From the point of view of applications, such a paradigm allows sending messages without using destination addresses. Instead, based on the interests of nodes defined through subscriptions to specific content, the network deduces the recipients of a message from its content and forwards this message as efficiently as possible to all relevant nodes. Publish / Subscribe systems have been initially defined for fixed networks but may also offer advantages for MANETs (Mobile Ad-Hoc Network - selfconfiguring networks of mobile nodes connected by wireless links) and VANETs (Vehicular Ad-Hoc Network, MANETs composed of vehicles). Innovative Applications for VANETs like accident or traffic jam information work naturally over a Publish / Subscribe system. In the same way, AANETs (Aeronautical Ad hoc NETworks which consist in MANETs with aircraft as nodes) can be seen as a subcategory of VANETs [1]. In the context of increasing air traffic and new needs in term of air ground communication systems such as the introduction of the 4D trajectory data link service in a near future [2], AANETs represent a relevant solution to supplement existing systems like satellite links. This solution does not need a heavy ground infrastructure and can offer an additional bit rate of several hundred of kbits/s for air/ground or air/air communications. Content based Publish / Subscribe application enjoys wide applicability in AANET where resources are limited compared to wired network. Notification of dangerous weather events (e.g.: storms, dust plumes due to volcanic activity) to aircraft whose trajectory comes close to the concerned geographical area is a good example of application.

Some Publish / Subscribe systems for VANETs, generally adapted to be aware of the geographical position of the node for the dissemination of events and subscriptions, have already been proposed. [1] establishes a survey of these solutions, categorizing them in three classes: geographic based routing solutions, proximity based routing solutions and overlay based solutions. The first two categories give good results in term of delivery ratio and network load for some specific applications but are too simplistic when events or subscriptions are applicable to wide geographical area. The third category of solutions, based on brokers to disseminate events and subscriptions in the network is the most promising to suit all kind of applications. However, in the context of large and highly dynamics network like AANETs, managing the connectivity and the synchronization between brokers imply that 


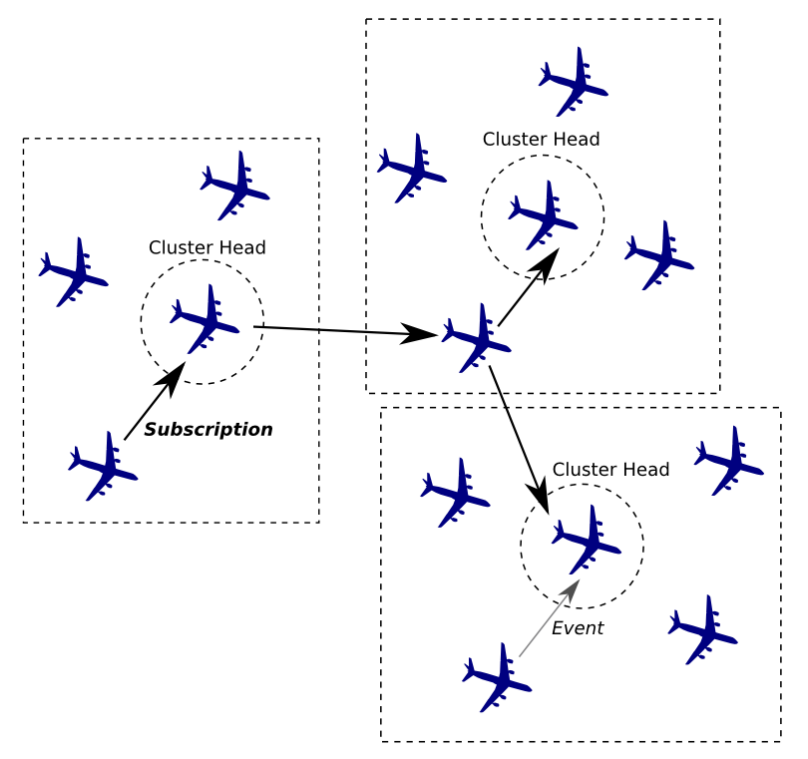

Figure 1. Principle of a Publish / Subscribe system over clusters

a large part of the bandwidth has to be dedicated to the overlay management. A solution to mitigate this problem and to offer a better scalability is to build a Publish / Subscribe system over a hierarchical structure such as a clustered structure. In a such a structure, the mobile nodes are organized in a number of virtual groups called clusters. Each cluster is composed of a cluster-head, serving as a local coordinator and in our case a broker for the Publish / Subscribe system, and regular nodes. Cluster-heads record all events sent by nodes belonging to their cluster and the subscriptions are sent to all cluster-heads using a multicast routing protocol as shown in Figure 1. When a cluster-head receives a subscription matching a recorded event, it sends it to the source of the subscription.

The efficiency of this kind of Publish / Subscribe system strongly depends on the performance of the clustering method and particularly on the stability of the clusters created. The aim of this article is to propose a new 1-Hop clustering algorithm dubbed CAPS (Cluster Algorithm for Publish / Subscribe) adapted to AANETs in terms of efficiency and stability. The next section presents the related work on this domain. Section details the CAPS algorithm. The validation of CAPS compared to other existing solutions is presented in Section. Finally, Section concludes this article and introduces further work on this topic.

\section{Related work}

Clustering algorithms have been proposed for MANET since a number of years. [3] proposes a survey and a classification for these solutions. A well known algorithm is LCC (Least Cluster Change) [4] which is an enhancement of Lowest ID Clustering (LIC) [5] or Highest Connectivity Clustering (HCC)[6]. To reduce the control overheads, LCC dissociates two phases: cluster formation and cluster maintenance. The latter being the key point since the modification of the cluster structure happens only when two cluster-heads move into communication range or when a mobile node looses its clusterhead. Another solution is 3hBAC (3-hop Between Adjacent Cluster-heads) [7] which forms a 1-hop non-overlapping cluster structure. To do so, $3 \mathrm{hBAC}$ introduces a new role, named cluster-guest which is used to avoid ripple effects in case of re-clustering.

Some other propositions use the mobility as first criteria to build the cluster structure. A good example is MOBIC [8] which supposes that nodes with low speed relative to their neighbours are the best candidate to become cluster-heads and thus proposes an aggregate local mobility metric for the cluster head election process. On-Demand WCA (Weighted Clustering Algorithm) [9] combines 4 metrics for this election: degree-difference, sum of the distance with all neighbours, average moving speed and clusterhead serving time. Another approach to minimize the control overhead consists in optimizing re-clustering conditions. $\alpha$-SSCA (Stability Structure Clustering Algorithm) [10] proposes to add a condition to merge clusters when two cluster-heads move into communication range of each other. One cluster-head gives up its role only if the stability factor (detailed in Section ) is below $\alpha$ whose value is between 0 and 1 .

VANETs being a subclass of MANETs with specific characteristics like constraints trajectory, specific clustering algorithms have been proposed for VANETs. They share the same aim which is to reduce the control overhead and use efficiently the distinctive features of VANETs. [11] proposes a survey on such techniques, many solutions it details are specific to VANETs composed by car (like MAC based clustering or Driver behavior clustering). However some solutions seem interesting for AANETs like a new stability-based clustering algorithm (SBCA) [12] which aims to reduce communication overhead and 
to increase the lifetime of the cluster. The election of a cluster-head is based on a merit value that combines the velocity difference between nodes and the number of neighbouring nodes. The specificity of this solution is the election of a secondary clusterhead which serves as a backup in case of problem with the primary cluster-head.

\section{The proposed clustering algorithm}

The main idea is to enhance solutions introduced in the previous section using efficiently the additional information available in AANETs nodes, especially its current position and speed. Therefore, as most of the 1-Hop clustering algorithm, this new solution is based on 3 steps: neighbours discovery, thanks to periodic hello messages, a procedure to elect the clusterhead at the beginning and a maintenance procedure. The key points of this new solution are the following.

- Hello messages contain more information, especially the current position and the current speed of the node.

- Elections of cluster-head are based on a combined-metric like WCA or SBCA with two metrics: one based on the number of neighbours, the other one based on relative speed.

- The maintenance procedure follows the $\alpha$-SSCA strategy, introducing two conditions before merging clusters. The first one is based on relative speed of cluster-heads and the second one is the same as $\alpha$-SSCA.

To go deeper in the cluster head election process, the first metric uses the number of neighbour to calculate a coefficient $D_{\text {coeff }}$ which is calculated thanks to the formula 1.

$$
D_{\text {coeff }}= \begin{cases}0 & \text { if } N<1 \\ \frac{N-1}{N_{L_{t h r e s h}}-1} & \text { if } 1 \leq N \leq N_{L_{\text {thresh }}} \\ 1 & \text { if } N_{L_{\text {thresh }}}<N \leq N_{H_{\text {thresh }}} \\ \left.1+\frac{N_{H_{\text {thresh }}}-N}{N_{L_{\text {thresh }}}}\right) & \text { if } N_{H_{\text {thresh }}}<N\end{cases}
$$

$N$ is the number of neighbour. $N_{L_{t h r e s h}}$ and $N_{H_{\text {thresh }}}$ are parameters of the algorithm. Figure 2 gives a graphical representation of this equation. This coefficient allows the selection of nodes whose the number of neighbour is between $N_{L_{\text {thresh }}}$ and $N_{H_{\text {thresh }}}$ to act as cluster-head. Hence, a good cluster-head

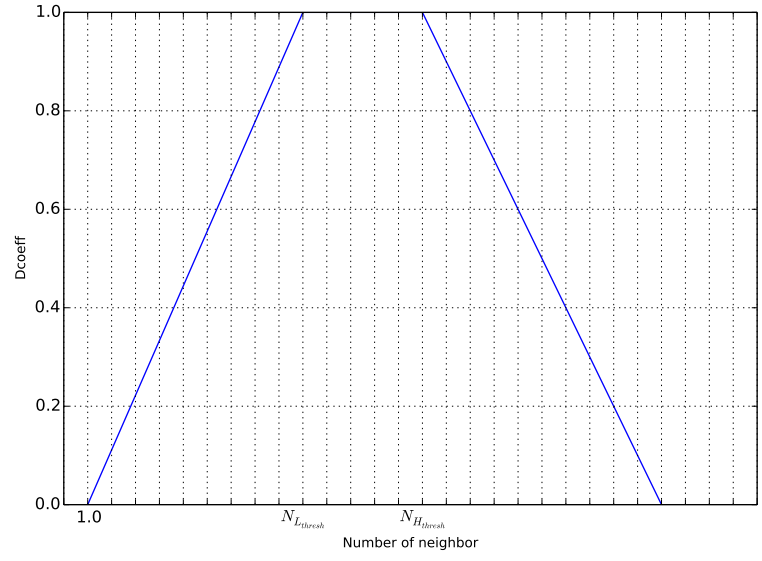

Figure 2. Calculation of $D_{\text {coeff }}$ (example with

$$
\left.N_{L_{\text {thresh }}}=10 \text { and } N_{H_{\text {thresh }}}=15\right)
$$

is a node with a minimum number of neighbour to reduce the number of clusters, yet with limited number of neighbours since, in our case, clusterheads have to record all the events of this group and all the active subscriptions of the network. Too many neighbours imply the management of a lots of events and subscriptions and thus some resources or synchronization issue when two clusters have to be merged. The formula 1 translates this consideration into a merit value for the election process. A study has been conducted to optimize the parameters of the algorithm which conclude to

$$
\left\{\begin{array}{l}
N_{L_{\text {thresh }}}=20 \\
N_{H_{\text {thresh }}}=25
\end{array}\right.
$$

for all the simulations detailed in the next section.

The second metric considers the node velocity. If, for a node $n, \overrightarrow{v_{n}}$ is the vector representing the velocity of the node and $N(n)$ represents the set of $n$ neighbours, then this metric is calculated thanks to the following equation.

$$
S_{\text {coeff }}=\sum_{i \in N(n)} \frac{\overrightarrow{v_{n}} \cdot \vec{v}_{i}}{\left\|\overrightarrow{v_{n}}\right\|\left\|\vec{v}_{i}\right\|}
$$

It selects preferably nodes which have a low relative velocity with respect to their neighbours. The weight used to elect cluster-heads is a combination of these two metrics.

$$
\text { Score }=\beta D_{\text {coeff }}+(1-\beta) S_{\text {coeff }}
$$

$\beta$ being chosen between 0 and 1 . The other important characteristic of this new algorithm is the condition 
used to determine whether two cluster-heads moving into communication range of each other need to merge their clusters. In such a case, one cluster-head has to give up its role if two conditions are fulfilled. The first one verifies that the two cluster-heads move in the same general direction. The speed vector is the best information to test this condition:

$$
\text { he } \vec{a} d 1 . h e \vec{a} d 2>0
$$

The second condition is the same as $\alpha$-SSCA algorithm. If $u$ and $v$ are two neighbour cluster-heads, $u$ gives its role up if

$$
\frac{\left|\left\{X_{i} \in C l(u) / X_{i} \in N(v)\right\}\right|}{|C l(u)|}>=\alpha
$$

Where $N(v)$ is the set of neighbours of $v$ and $C l(u)$ represents the set of cluster members of $u$.

\section{Validation}

In order to validate our new clustering algorithm, a dedicated simulation model has been developed on OMNET++ ([13]). We also implemented three others 1-Hop clustering algorithms to assess the performances of our new solution: LCC-HCC, $\alpha$-SSCA (with $\alpha$ equals to 0.5 ) and MOBIC.

\section{Scenario}

These 4 clustering algorithms have been compared in an modeled AANET context based on real air traffic traces taken from Eurocontrol. As AANETs are especially interesting in areas where no ground infrastructures are available, as over oceans, we choose to focus on the north transatlantic traffic. Therefore, our simulations are taking into account aircraft whose latitude are in the $\left[23,5^{\circ} \mathrm{N}-70^{\circ} \mathrm{N}\right]$ interval and the longitude belongs to the $\left[90^{\circ} \mathrm{W}-10^{\circ} \mathrm{E}\right]$ interval. Furthermore, in order to evaluate the solutions in different situations, the traffic of several days has been used as input for the simulations with similar results. Since most of the north transatlantic traffic is composed by regular flights, any two days close on the calendar are very similar in terms of number of aircraft and trajectories. The difference lies mainly in the period of the year. For example, on average, there is much more traffic during the summer. That's why, in this article, we choose to present the results for two days. The first one (2012/12/25) is a day where the commercial traffic is very low as opposed to the second one $(2012 / 10 / 05)$.
Table I. Simulations parameters

\begin{tabular}{|l|c|}
\hline Duration of the simulation & $24 \mathrm{~h}$ \\
\hline Day & $2012 / 12 / 25$ and $2012 / 10 / 05$ \\
\hline Transmission range & $300 \mathrm{~km}$ \\
\hline Physical layer bitrate & $1 \mathrm{Mb} / \mathrm{s}$ \\
\hline Hello interval & $1 \mathrm{~second}$ \\
\hline
\end{tabular}

Table I summarizes the configuration used for the simulations. The layer 2 protocol has been simulated using the IdealWireless module form INET 1 with fixed transmission range and bit rate. However the values used for the simulations are deduced from a previous study evaluating the feasibility of AANETs with commercial aircraft over the north transatlantic corridor. In particular, [14] evaluates the use of CDMA in the layer 2 to interconnect aircraft. Depending on the configuration such as the used bandwidth or modulation, the obtained link capacity between aircraft varies from hundreds of Kbits/s to two Mbits/s for a transmission range of several hundred of kilometers. The choice of one Mbits/s is a good trade off for our simulations.

To evaluate the efficiency of the clustering algorithms, three parameters are measures during the simulations:

1) The number of cluster-head at a given time.

2) The total number of cluster-head changes, a lower value indicates a better stability of the clustering algorithm.

3) The lifetime of cluster-heads which is also an indicator of the cluster stability.

\section{Results}

Figures 3 and 4 show all the results obtained from the simulations. To facilitate the analysis, the cluster head duration is plotted as a cumulative distribution probability. In the same way, the first graph represents the number of aircraft during the considered day to easily make a correlation between this number and the number of cluster head. As expected, most of the traffic in the North Atlantic Airspace is organized in two major flows (peaks on the graph): a westbound flow departing Europe in the morning and an eastbound flow departing North America in the evening (UTC time).

\footnotetext{
${ }^{1}$ http://inet.omnetpp.org/
} 

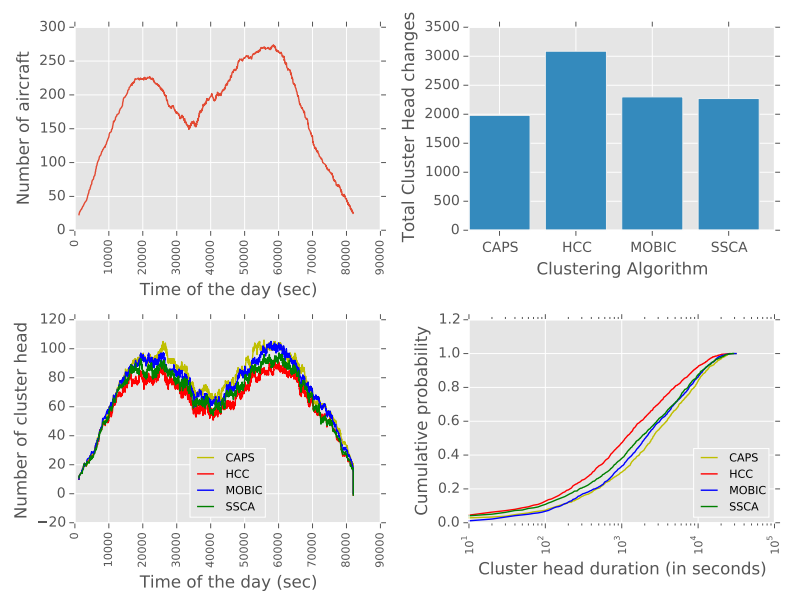

Figure 3. Results for the December 252012
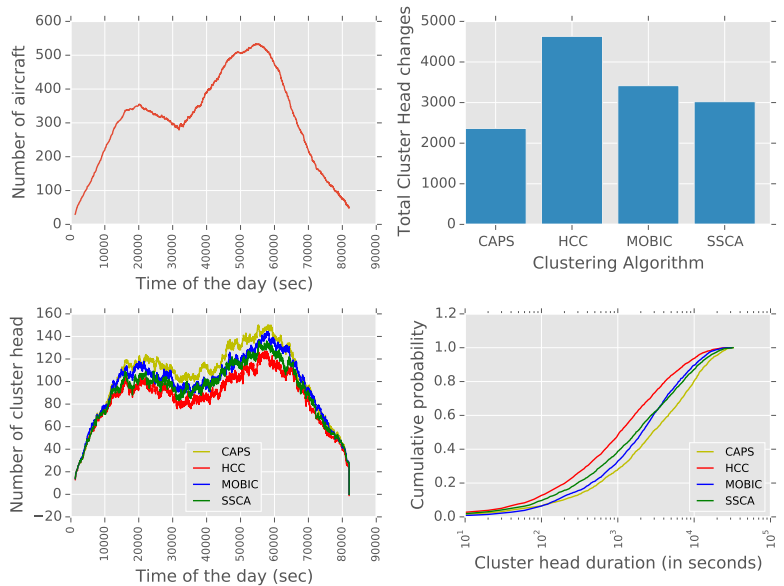

Figure 4. Results for the October 052012

The results show that the clustering algorithms taking into account the mobility of nodes offer better stability since the cluster-head duration is higher. The counterpart is a higher number of cluster head. In detail, CAPS gives the best performance compared to others solutions in terms of stability. Table II shows that the mean lifetime of clusters for CAPS is $10 \%$ higher than for MOBIC while the number of clusterheads stays close to the MOBIC one. CAPS seems to be a solid basis for a Publish /Subscribe system adapted to AANETs since it will offer a very stable overlay.

\section{Conclusion}

In this article, after emphasizing the interest of a cluster structure as an overlay of an efficient VANET adapted Publish / Subscribe systems, a brief
Table II. Detailed results for the 2012/12/25

\begin{tabular}{|l|c|c|}
\hline & CAPS & MOBIC \\
\hline Number of cluster-heads (mean) & 80.7 & 78.9 \\
\hline Cluster-head duration (mean) & $4775 \mathrm{~s}$ & $4152 \mathrm{~s}$ \\
\hline Number of cluster-head changes & 1981 & 2298 \\
\hline
\end{tabular}

survey on clustering algorithms adapted to MANETs is proposed, focused on low maintenance algorithm. While interesting, these solutions don't use all the information available to the nodes in an AANET. Particularly, to exploit the velocity of aircraft in addition to the number of neighbours, we created a new clustering algorithm, and then compared it to other solutions through simulations on OMNET++ with a realistic AANET mobility model. Simulation results show that this new algorithm provides better performance than other simulated solutions in terms of stability. It minimizes the number of cluster-head changes and increases the lifetime of clusters. This new algorithm represents a solid basis for a hierarchical Publish / Subscribe systems adapted to AANET.

Our further work will concentrate on the OMNET++ implementation of this Publish / Subscribe system and its evaluation in comparison to other solutions like proximity or geographic routing based Publish / Subscribe.

\section{References}

[1] M. Royer, A. Pirovano, and F. Garcia, "Survey on Context-Aware Publish/Subscribe Systems for VANET", in Communication Technologies for Vehicles, ser. Lecture Notes in Computer Science, vol. 7865, Springer Berlin Heidelberg, 2013, pp. 4658, ISBN: 978-3-642-37973-4. DOI: 10.1007/978-3642-37974-1_4. [Online]. Available: http://dx.doi. org/10.1007/978-3-642-37974-1_4.

[2] M. Jackson, J. Gonda, R. Mead, and G. Saccone, "The 4D trajectory data link (4DTRAD) service Closing the loop for air traffic control", in Integrated Communications, Navigation and Surveillance Conference, 2009. ICNS '09., 2009, pp. 1-10. DOI: 10. 1109/ICNSURV.2009.5172860.

[3] J. Yu and P. Chong, "A survey of clustering schemes for mobile ad hoc networks", Communications Surveys Tutorials, IEEE, vol. 7, no. 1, pp. 32-48, 2005, ISSN: 1553-877X. DOI: 10.1109/COMST.2005. 1423333 . 
[4] C.-C. Chiang, H.-K. Wu, W. Liu, and M. Gerla, "Routing in clustered multihop, mobile wireless networks with fading channel", in proceedings of IEEE SICON, vol. 97, 1997, pp. 197-211.

[5] A. Ephremides, J. Wieselthier, and D. Baker, "A design concept for reliable mobile radio networks with frequency hopping signaling", Proceedings of the IEEE, vol. 75, no. 1, pp. 56-73, 1987, ISSN: 00189219. DOI: 10.1109/PROC.1987.13705.

[6] A. Ephemrides, J. E. Wieselthier, and D. J. Baker, "A Design Concept for Reliable Mobile Radio Networks with Frequency Hopping Signaling", in Proceedings of the IEEE 75 (1), 1987, pp. 56-73.

[7] J. Yu and P. H. J. Chong, "3hBAC (3-hop between adjacent clusterheads): a novel non-overlapping clustering algorithm for mobile ad hoc networks", in Communications, Computers and signal Processing, 2003. PACRIM. 2003 IEEE Pacific Rim Conference on, vol. 1, 2003, 318-321 vol.1. DOI: 10 . 1109/ PACRIM.2003.1235781.

[8] P. Basu, N. Khan, and T. D. C. Little, "A mobility based metric for clustering in mobile ad hoc networks", in Distributed Computing Systems Workshop, 2001 International Conference on, 2001, pp. 413418. DOI: 10.1109/CDCS.2001.918738.

[9] M. Chatterjee, S. K. Das, and D. Turgut, "An on-demand weighted clustering algorithm (WCA) for ad hoc networks.", in GLOBECOM, IEEE, 2000, pp. 1697-1701, ISBN: 0-7803-6451-1. [Online]. Available: http : / / dblp . uni - trier . de / db / conf / globecom / globecom2000 . html \# ChatterjeeDT00 ; http : / / dx . doi . org / 10 . 1109 / GLOCOM . 2000 . 891926 ; http : / / www . bibsonomy . org / bibtex / 2717dd108e75bdeb64064fba936f8a82a/dblp.
[10] B. Guizani, B. el Ayeb, and A. Koukam, "Hierarchical cluster-based link state routing protocol for large self-organizing networks.", in HPSR, IEEE, 2011, pp. 203-208. [Online]. Available: http : / / dblp . uni - trier . de / db / conf / hpsr / hpsr2011 . html \# GuizaniAK11; http://dx . doi . org/10.1109/HPSR . 2011.5986027 ; http://www. bibsonomy. org/bibtex / 2edd157b8e17592f87dc61132582ba0d9/dblp.

[11] R. S. Bali, N. Kumar, and J. J. Rodrigues, "Clustering in vehicular ad hoc networks: taxonomy, challenges and solutions", Vehicular Communications, 2014.

[12] A. Ahizoune and A. Hafid, "A new stability based clustering algorithm (SBCA) for VANETs.", in LCN Workshops, IEEE, 2012, pp. 843-847, ISBN: 978-1-4673-2130-3. [Online]. Available: http://dblp. uni - trier . de / db / conf / lcn / lcnw2012 . html \# AhizouneH12.

[13] A. Varga and R. Hornig, "An overview of the OMNeT++ simulation environment", in Simutools '08: Proceedings of the 1st international conference on Simulation tools and techniques for communications, networks and systems \& workshops, Marseille, France: ICST (Institute for Computer Sciences, Social-Informatics and Telecommunications Engineering), 2008, pp. 1-10, ISBN: 978-963-979920-2.

[14] F. Besse, A. Pirovano, F. Garcia, and J. Radzik, "Interference estimation in an aeronautical ad hoc network", in Digital Avionics Systems Conference (DASC), 2011 IEEE/AIAA 30th, 2011, pp. 4C6-14C6-11. 